\title{
Correlation of functional claudication distance with canal diameter in patients with lumbar canal stenosis: a preliminary report
}

\author{
DOI: https://doi.org/10.5114/pq.2021.105755
}

\author{
Gurjant Singh ${ }^{1,2 \oplus}{ }^{\oplus}$, Sunita Sharma1', Parveen Kumar', Manu Goyal', Asir John Samuel' \\ ${ }^{1}$ Maharishi Markandeshwar Institute of Physiotherapy and Rehabilitation, Maharishi Markandeshwar \\ (Deemed to be University), Mullana, India \\ 2 Department of Physiotherapy, University Institute of Applied Health Sciences, Chandigarh University, Mohali, India
}

\begin{abstract}
Introduction. Neurogenic claudication is the classic clinical presentation of patients with lumbar canal stenosis. Several studies have addressed the assessment of such patients, but the impact of such change in canal diameter on the distance of walking is still to be identified. The study aimed to assess the functional claudication distance in relation to the canal diameter change in patients with lumbar canal stenosis.

Methods. Overall, 50 patients presenting with lumbar canal stenosis with the canal diameter of 8-12 $\mathrm{mm}$ at the level of $\mathrm{L} 4$ and below were recruited for this study. Individuals with trauma or associated fracture, laminectomy, or congenital stenosis were excluded. The demographic characteristics and anthropometrics of each patient were established. Canal diameter was documented with the help of magnetic resonance imaging. For functional claudication distance measurement, the participants were asked to walk on a treadmill up to the distance at which they preferred to stop owing to pain.

Results. The mean \pm standard deviation $(95 \% \mathrm{Cl})$ of canal diameter and functional claudication distance were found to be $10.33 \pm 1.26 \mathrm{~mm}(10.00-10.69)$ and $141.29 \pm 44.20 \mathrm{~m}$ (130-153.44), respectively. The Pearson correlation coefficient determined a moderate to good positive correlation $(r=0.73)$ between lumbar canal diameter and functional claudication distance.

Conclusions. The study concluded that an increase in the anteroposterior canal diameter might increase functional claudication distance or vice versa. The results may be helpful in estimating the distance of walking in patients with lumbar canal stenosis. Key words: lumbosacral region, magnetic resonance imaging, pain, stenosis, treadmill
\end{abstract}

\section{Introduction}

Lumbar canal stenosis (LCS) is defined as a condition that comprises narrowing of the central canal due to posterior intervertebral disc protrusion, spondylolisthesis, ligamentum flavum hypertrophy and buckling, facet joint degeneration, or degenerative disc bulging, causing the compression of nerve roots [1, 2]. The normal diameter of the lumbar spinal canal ranges between 15 and $27 \mathrm{~mm}$; a diameter less than $12 \mathrm{~mm}$ is considered as stenosis [3]. Magnetic resonance imaging (MRI) is routinely used in clinical practice, being the modality of choice to diagnose LCS [4]. Neurogenic claudication (NC) is one of the cornerstone features of LCS; it is induced by a discomfort and pain in buttocks, legs, and thighs and is indubitably a reason for spinal surgeries like laminectomy among individuals with stenosis $[5,6]$. Such surgeries are praiseworthy but for a limited number of patients [7], and are usually avoided by patients. Symptoms of NC are exacerbated while walking and standing and are eased by sitting or bending forward [8]. All these symptoms adversely affect the activities of daily living and have a negative impact on quality of life [9], forcing patients to use analgesics [10].

Anatomically, spinal stenosis can occur centrally, within the lateral recess, at the level of the intervertebral disc, or in the foramen. The radiological criteria for lumbar spinal stenosis depend on the location of stenosis in MRI; central canal stenosis is diagnosed with the anteroposterior diameter of the spinal canal $\leq 12 \mathrm{~mm}$ [11], and compression of the traversing nerve root occurs in lateral recess stenosis [12]. LCS can be congenital or acquired; it can also result from a combination of congenital and acquired factors. In the congenital type, the aetiology is the presence of short pedicles as in achondroplasia, and the acquired type primarily involves a degenerative disease [13]. The acquired type is caused by age-related degenerative changes that cause narrowing of the canal and lead to compression of nerve roots [14]. Owing to pain in back and thighs, the patient adopts a protective strategy while walking to limit spinal movements, which decreases the distance of walking [15]. The distance at which the patient stops walking because of claudication pain is defined as functional claudication distance (FCD) and constitutes a reliable method to measure functional impairment in NC [16]. Studies have reported a decreased distance of walking among patients with LCS, but there is no standardized method followed to evaluate that distance; the same has been noted with the self-paced walking test [17].

To mark the FCD, a treadmill could be used as a standardized method [18]. Then, the standard protocol for treadmill walking is required to be followed. It includes the speed of walking over the treadmill, the angle of inclination, and the demonstration of the way of walking and stabilization over the treadmill. For patients with claudication pain, a speed of $3.2 \mathrm{~km} / \mathrm{h}$ is recommended [16]. As for the way of walking over a treadmill, a demonstration video could be applied. To the best of the authors' knowledge, there was a scarcity of literature regarding the distance of walking (with tolerable pain) with relation to the diameter of the spinal canal in NC cases. This study aimed at finding the effect of the canal diameter change on FCD. A change in the lumbar canal diameter may have a positive or negative impact on FCD.

Correspondence address: Gurjant Singh, Department of Physiotherapy, University Institute of Applied Health Sciences, Chandigarh University, NH-95, Ludhiana - Chandigarh State Hwy, Mohali, Punjab 140413, India, e-mail: singh.guri547@gmail.com, https://orcid.org/0000-0002-7421-8868 


\section{Subjects and methods}

\section{Patients and design}

The study flowchart is presented in Figure 1. The investigation was conducted in the period from October 2018 to September 2019 in a physiotherapy department research laboratory. Patients were recruited from the spine clinic of a tertiary care teaching hospital on the basis of the objective of the study, with the purposive sampling method. Individuals with a complaint of backache were screened $(n=105)$ by an experienced orthopaedic surgeon for possible inclusion in the study. The patients presenting with LCS due to spondylolisthesis, posterior intervertebral disc protrusion, ligamentum flavum hypertrophy and buckling, facet joint degeneration, or degenerative disc bulging [19] were included in the study. Those with any history of trauma or associated fracture, laminectomy, or congenital spinal deformity or stenosis were excluded. Individuals with body mass index (BMI) $\geq 30$ were also excluded from the study as they fell in the obese category according to the BMI classification by the World Health Organization, and obesity could be another factor responsible for a decreased walking distance [20, 21]. The patients with symptoms of NC were asked by the orthopaedic surgeon for MRI investigation before referring them for inclusion in the study. MRI was performed by an experi- enced radiologist, blinded to the FCD of each patient, with the use of a 1.5T Philips Multiva MRI system. The imaging sequences included were: sagittal $T_{1}$, sagittal $T_{2}$, coronal short tau inversion recovery (STIR), axial $T_{1}$, and axial $T_{2}$. On MRI, 50 patients with a canal diameter of 8-12 $\mathrm{mm}$ at the level of $L_{4}$ and below were recruited for the study. Demographic characteristics such as name (entered as code), age, sex, and occupation, as well as anthropometric measures, such as weight and height of each patient, were noted. Height was determined with a measuring tape and weight with calibrated digital scales. BMI was calculated with the standard formula:

$$
\mathrm{BMI}=\text { weight }(\mathrm{kg}) / \text { height }^{2}\left(\mathrm{~m}^{2}\right)
$$

The central canal diameter was recorded with the help of MRI. Being the gold standard for measuring the walking distance, a treadmill was used to determine FCD [18]. A demonstration video of walking on a treadmill was shown to the patients to teach them how to perform the activity. After the demonstration, they were asked to walk on a treadmill, with the speed gradually increased to $3.2 \mathrm{~km} / \mathrm{h}$ [16] in the initial 30 seconds and kept constant thereafter, at $0^{\circ}$ inclination, under the supervision of a physiotherapist. The subjects were asked to walk as far as possible. The distance at which they preferred to stop because of pain (FCD) was noted for each patient.

Patients assessed by physical screening and magnetic resonance imaging $(n=76)$

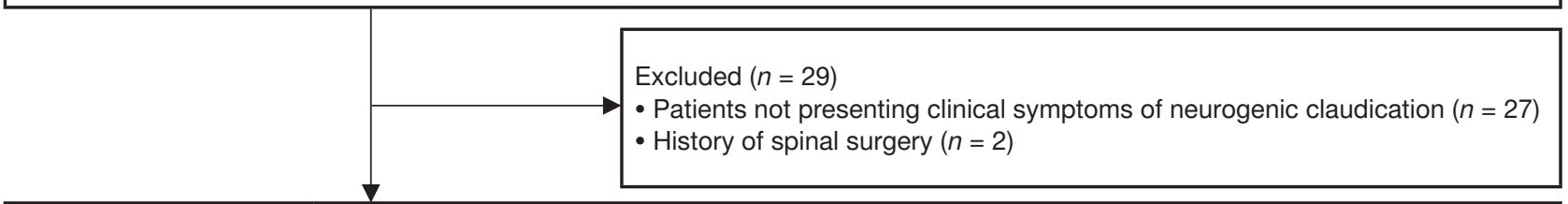

Patients with central canal stenosis with canal diameter of 8-12 $\mathrm{mm}$ at the level of $\mathrm{L}_{4}$ and below were recruited for the inclusion in the study $(n=50)$

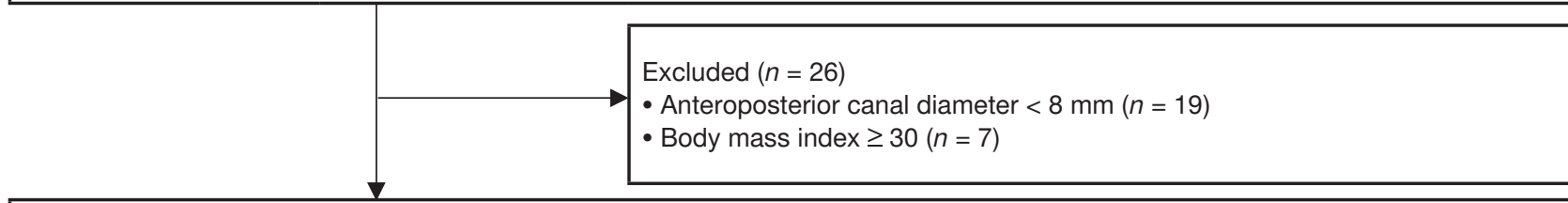

Informed consent was obtained from each patient for voluntarily participation in the study

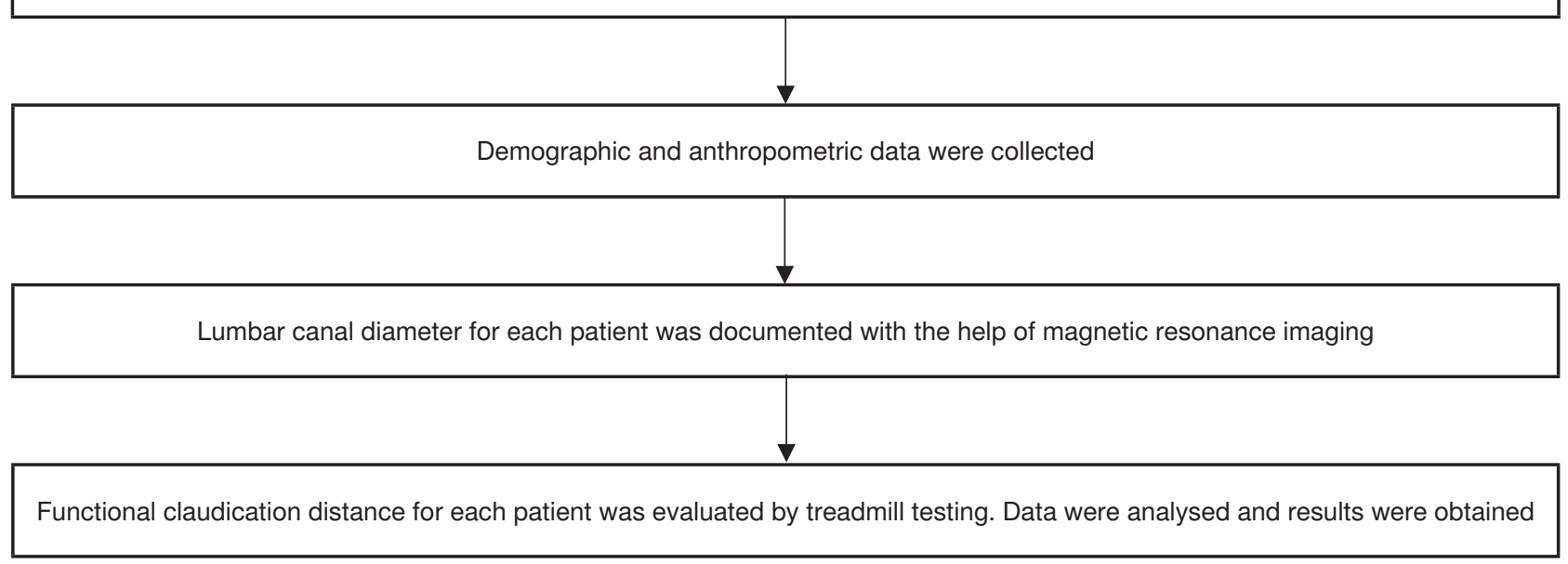

Figure 1. Flowchart of the procedure 


\section{Data analysis}

The sample size was determined with the sample size calculation formula:

$$
n=[(Z \alpha+Z \beta) / C]^{2}+3
$$

where $Z \alpha$ is the standard normal deviate for $\alpha, Z \beta$ is the standard normal deviate for $\beta$, and $C$ was calculated as:

$$
\mathrm{C}=0.5 \times \ln [(1+r) /(1-r)]
$$

The value of the correlation coefficient $(r=0.47)$ was derived from a previous study [22]. Thus, by using the abovementioned sample size calculation formula, a minimum sample size was calculated as $n=33$. With the consideration of the footfall of the patients and the minimum calculated sample size, 50 patients were recruited in the study.

The data were analysed with the Statistical Package for the Social Sciences (SPSS) version 20 software. The normality of data distribution was established with the KolmogorovSmirnov test $(n=50)$. As the data were normally distributed, demographic and descriptive characteristics of all patients were expressed as mean (95\% confidence interval [Cl]). The Pearson correlation coefficient parametric test was used to find the correlation between canal diameter and FCD.

\section{Ethical approval}

The research related to human use has complied with the national ethical guidelines for biomedical and health research involving human subjects (revised 2017) [23], has followed the tenets of the Declaration of Helsinki, and has been approved by the student project committee and ethical

Table 1. Demographic characteristics of participants

\begin{tabular}{|l|c|}
\hline Variable & Mean $\pm S D(95 \% \mathrm{Cl})$ \\
\hline Age (years) & $36.78 \pm 7.23(34.72-38.83)$ \\
\hline Height $(\mathrm{cm})$ & $165.8 \pm 8.15(163.5-168.12)$ \\
\hline Weight $(\mathrm{kg})$ & $68.8 \pm 12.80(65.16-72.43)$ \\
\hline Body mass index $\left(\mathrm{kg} / \mathrm{m}^{2}\right)$ & $24.95 \pm 3.98(23.82-26.1)$ \\
\hline
\end{tabular}

Table 2. Descriptive statistics of the variables

\begin{tabular}{|l|c|}
\hline Variable & Mean $\pm S D(95 \% \mathrm{Cl})$ \\
\hline Canal diameter $(\mathrm{mm})$ & $10.33 \pm 1.26(10.00-10.69)$ \\
\hline Functional claudication distance $(\mathrm{m})$ & $141.29 \pm 44.20(130-153.44)$ \\
\hline
\end{tabular}

committee of Maharishi Markandeshwar Institute of Physiotherapy and Rehabilitation, Mullana, Haryana, India (MMDU/ IEC/1181).

\section{Informed consent}

Informed consent has been obtained from all individuals included in this study.

\section{Results}

The study involved 50 patients: 29 males and 21 females $(n=21)$. The participants' mean \pm standard deviation $(95 \% \mathrm{Cl})$ age was 36 years 9 months \pm 7 years 4 months (34 years 9 months-38 years 10 months), as shown in Table 1 . The mean \pm standard deviation $(95 \% \mathrm{Cl})$ canal diameter and FCD equalled $10.33 \pm 1.26 \mathrm{~mm}(10.00-10.69)$ and $141.29 \pm$ $44.20 \mathrm{~m}$ (130-153.44), respectively, as presented in Table 2. The demographic and descriptive statistics of male and female patients are depicted in Table 3. Statistically significant differences were found in height and weight between male and female patients, but not in any other variables. The correlation between canal diameter and FCD was measured by using the Pearson correlation coefficient. A statistically significant positive correlation was found $\left(r=0.73, R^{2}=0.527\right.$, $p<0.001$ ), as indicated in Table 4 and Figures 2 and 3 . The prediction equation to estimate the canal diameter (CD) was found to be as follows:

$$
\mathrm{CD}(\mathrm{mm})=7.413+0.021 \mathrm{FCD}
$$

The value of correlation coefficient $(r)$ was interpreted as follows:

- below -0.75: good to excellent negative correlation;

- from -0.50 to -0.75 : moderate to good negative correlation;

- from -0.25 to -0.50 : fair negative correlation;

-0 : no correlation;

- 0.25-0.50: fair positive correlation;

- 0.50-0.75: moderate to good positive correlation;

- above 0.75: good to excellent positive correlation.

Table 4. Correlation between canal diameter and functional claudication distance

\begin{tabular}{|l|c|c|}
\hline Variable & $r$ & $p$ \\
\hline Functional claudication distance & 0.73 & $<0.001^{*}$ \\
\hline
\end{tabular}

${ }^{*}$ significant $(p \leq 0.05)$

\begin{tabular}{|c|c|c|c|}
\hline \multirow{2}{*}{ Variable } & \multicolumn{2}{|c|}{ Mean $\pm S D(95 \% \mathrm{Cl})$} & \multirow{2}{*}{$p$} \\
\hline & Males $(n=29)$ & Females $(n=21)$ & \\
\hline Age (years) & $37.41 \pm 7.64(34.50-40.32)$ & $35.90 \pm 6.70(32.85-38.96)$ & 0.47 \\
\hline Height (cm) & $168.94 \pm 8.10(165.85-172.02)$ & $161.48 \pm 6.09(158.71-164.25)$ & $<0.01^{*}$ \\
\hline Weight (kg) & $73.17 \pm 13.20(68.15-78.19)$ & $62.76 \pm 9.55(58.41-67.11)$ & $<0.01^{*}$ \\
\hline Body mass index $\left(\mathrm{kg} / \mathrm{m}^{2}\right)$ & $25.57 \pm 4.15(24.00-27.14)$ & $24.11 \pm 3.64(22.46-25.77)$ & 0.20 \\
\hline Canal diameter (mm) & $10.34 \pm 1.77(9.90-10.79)$ & $10.32 \pm 1.40(9.69-10.96)$ & 0.97 \\
\hline Functional claudication distance (m) & $144.58 \pm 43.91(127.88-161.29)$ & $136.77 \pm 45.25(116.16-157.36)$ & 0.54 \\
\hline
\end{tabular}

Table 3. Demographic and descriptive statistics in males and females 


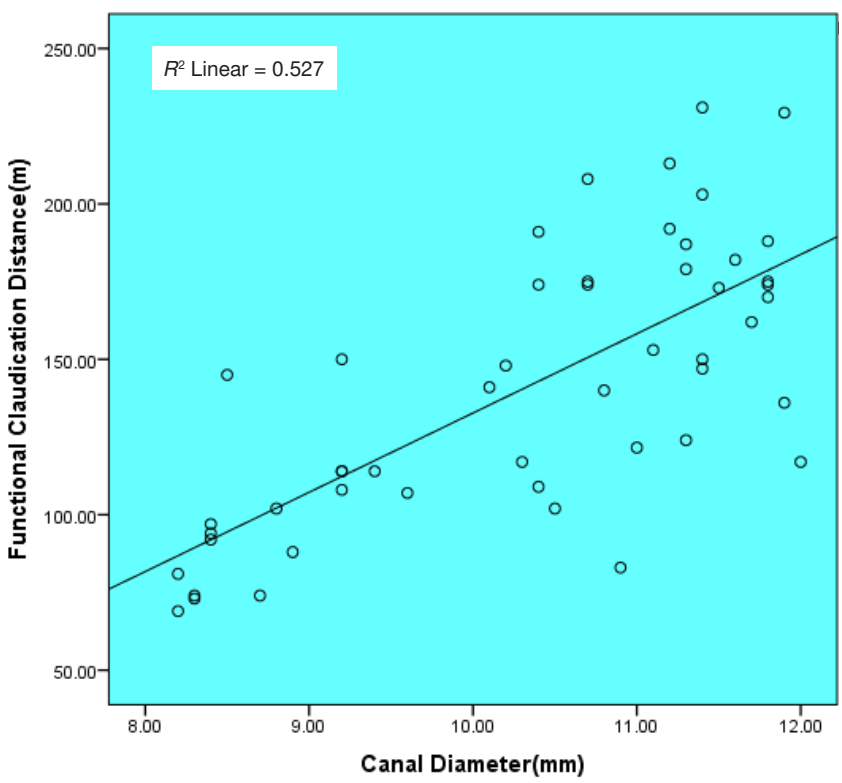

Figure 2. The scatter diagram displaying the level of correlation between canal diameter and functional claudication distance

\section{Discussion}

The present study revealed a moderate to good degree of positive correlation (Portney and Watkins criteria) [24] between the canal diameter and FCD $(r=0.73)$, as shown in Table 4. The strong relationship may be attributed to the familiarization of the patients with treadmill walking. FCD was found to be a relevant and absolute measure to determine functional capacity in intermittent claudication patients, as reported by Kruidenier et al. [16]. Those authors also observed a strong positive correlation between FCD and disease severity. The results of their study are in line with those of the present study in terms of FCD. However, the patient population in both papers are different. The independent variable (canal diameter) accounts for $52.7 \%$ of variability of the dependent variable (FCD). If one substitutes the mean value of FCD in the prediction equation, this study implies that a 1-mm increase in canal diameter adds $10.38 \mathrm{~m}$ of walking.

The present study was a preliminary one and included patients with mild to moderate LCS exhibiting symptoms of NC, with limited variations in age. The prediction equation formulated in this study was based on the study findings, which limits extrapolation. It is not the authors' aim to recommend the prediction equation for the evaluation of LCS patients: $\mathrm{MRI}$ and clinical findings are the only reliable diagnostic measures as per the literature.

The present study might be helpful clinically in the prognosis of the condition and to obtain an estimated pain-free walking distance on the basis of the lumbar canal diameter. A conservative treatment may be planned with the consideration of FCD so as to avoid exertion beyond the proposed distance in designing a home protocol for the patients or to implement a period of rest after specific exertion.

Future research could be conducted among patients with a smaller canal diameter, including asymptomatic individuals with LCS, as well as in different age groups. An interventional study can also be planned to find the efficacy of physiotherapeutic manual therapy techniques in improving FCD in patients with LCS.

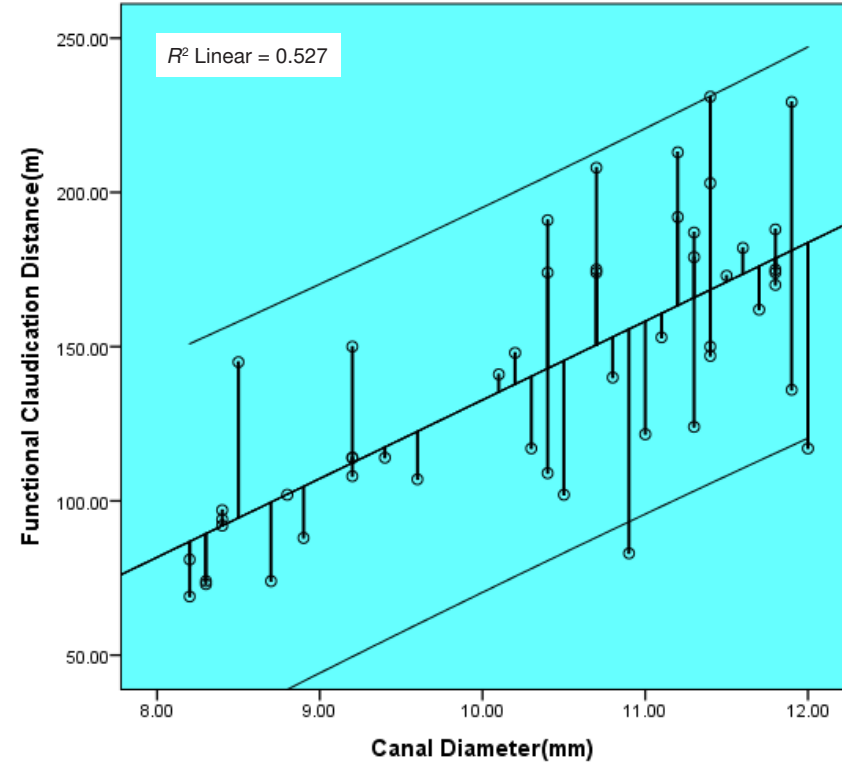

Figure 3. Deviant scores from least-square regression line between canal diameter and functional claudication distance

\section{Limitations}

There are several limitations to the present study. It was a single-centre study and included only patients with mild to moderate LCS. The age variations were also limited. The results may vary in a higher age group as more degenerative changes could be seen in such individuals.

\section{Conclusions}

This study implies that there was a moderate to good positive correlation between canal diameter and FCD. That signifies that an increase in the canal diameter may raise FCD or vice versa in LCS patients with NC.

\section{Availability of data and materials}

The datasets used and analysed during the current study are available from the corresponding author on reasonable request.

\section{Funding}

This research received no specific grant from any funding agency in the public, commercial, or not-for-profit sectors.

\section{Disclosure statement}

No author has any financial interest or received any financial benefit from this research.

\section{Conflict of interest}

The authors state no conflict of interest.

\section{References}

1. Azimi P, Mohammadi HR, Benzel EC, Shahzadi S, Azhari S. Lumbar spinal canal stenosis classification criteria: a new tool. Asian Spine J. 2015;9(3):399-406; doi: 10.4184/asj.2015.9.3.399.

2. Fritz JM, Delitto A, Welch WC, Erhard RE. Lumbar spinal stenosis: a review of current concepts in evaluation, management, and outcome measurements. Arch Phys Med Rehabil. 1998;79(6):700-708; doi: 10.1016/S0003 -9993(98)90048-X. 
3. Anasuya DG, Jayashree A, Moorthy NLN, Madan S. Anatomical study of lumbar spinal canal diameter on MRI to assess spinal canal stenosis. Int J Anat Res. 2015;3(3): 1441-1444; doi: 10.16965/ijar.2015.261.

4. De C, Ray MK, Chatterjee B, Duttaroy S, Ghosh PK, De C. Correlation between clinical and imaging finding of symptomatic degenerative lumbar spine disease. Saudi J Sports Med. 2018;18(2):79-84; doi: 10.4103/sjsm. sjsm_24_17.

5. Ju J-H, Ha H-G, Jung C-K, Kim H-W, Lee C-Y, Kim J-H. Patterns of epidural venous varicosity in lumbar stenosis. Korean J Spine. 2012;9(3):244-249; doi: 10.14245/ kjs.2012.9.3.244.

6. Kim K, Shin K-M, Hunt CL, Wang Z, Bauer BA, Kwon O, et al. Nonsurgical integrative inpatient treatments for symptomatic lumbar spinal stenosis: a multi-arm randomized controlled pilot trial. J Pain Res. 2019;12:11031113; doi: 10.2147/JPR.S173178.

7. Mbada CE, Makinde MO, Odole AC, Dada OO, Ayanniyi O, Salami AJ, et al. Comparative effects of clinic- and virtual reality-based McKenzie extension therapy in chronic non-specific low-back pain. Hum Mov. 2019;20(3):6679; doi: 10.5114/hm.2019.83998.

8. Comer CM, Redmond AC, Bird HA, Conaghan PG. Assessment and management of neurogenic claudication associated with lumbar spinal stenosis in a UK primary care musculoskeletal service: a survey of current practice among physiotherapists. BMC Musculoskelet Disord. 2009;10:121; doi: 10.1186/1471-2474-10-121.

9. Otani K, Kikuchi S, Yabuki S, Igarashi T, Nikaido T, Watanabe $\mathrm{K}$, et al. Lumbar spinal stenosis has a negative impact on quality of life compared with other comorbidities: an epidemiological cross-sectional study of $1862 \mathrm{com}-$ munity-dwelling individuals. Sci World J. 2013;2013: 590652; doi: 10.1155/2013/590652.

10. Kamińska J, Pawlak M. The use of analgesics among Poznań marathon runners. Hum Mov. 2019;20(3):23-28; doi: 10.5114/hm.2019.83993.

11. Steurer J, Nydegger A, Held U, Brunner F, Hodler J, Porchet $F$, et al. LumbSten: the lumbar spinal stenosis outcome study. BMC Musculoskelet Disord. 2010;11:254; doi: 10.1186/1471-2474-11-254.

12. Rajagopal TS, Marshall RW. Understanding and treating spinal stenosis. J Bone Joint Surg (Br). 2010;1-7.

13. Ahmad T, Goel $P$, Babu CSR. A study of lumbar canal by M.R.I. in clinically symptomatic and asymptomatic subjects. J Anat Soc India. 2011;60(2):184-187; doi: 10.1016/ S0003-2778(11)80022-5.

14. Ammendolia $\mathrm{C}$, Chow N. Clinical outcomes for neurogenic claudication using a multimodal program for lumbar spinal stenosis: a retrospective study. J Manipulative Physiol Ther. 2015;38(3):188-194; doi: 10.1016/j.jmpt. 2014.12.006.

15. Łozińska P, Wójtowicz D, Wdowiak P, Dziuba-Słonina A. Changes in kinematic parameters during walking in adults with low back pain subjected to Vojta therapy. A pilot study. Physiother Quart. 2019;27(2):22-28; doi: 10.5114/pq.2019.84273.

16. Kruidenier LM, Nicolaï SPA, Willigendael EM, de Bie RA, Prins $\mathrm{MH}$, Teijink JAW. Functional claudication distance: a reliable and valid measurement to assess functional limitation in patients with intermittent claudication. BMC Cardiovasc Disord. 2009;9:9; doi: 10.1186/1471-2261-9-9.

17. Ammendolia $C$, Côté $P$, Southerst $D$, Schneider M, Budgell B, Bombardier $\mathrm{C}$, et al. Comprehensive nonsurgical treatment versus self-directed care to improve walking ability in lumbar spinal stenosis: a randomized trial. Arch Phys Med Rehabil. 2018;99(12):2408-2419.e2; doi: 10.1016/j.apmr.2018.05.014.

18. Le Faucheur A, Abraham $P$, Jaquinandi V, Bouyé $P$, Saumet JL, Noury-Desvaux B. Measurement of walking distance and speed in patients with peripheral arterial disease: a novel method using a global positioning system. Circulation. 2008;117(7):897-904; doi: 10.1161/CIRCULATIONAHA.107.725994.

19. Kumar S, Narkeesh A. Effect of integrated exercise protocol in lumbar spinal stenosis as compare with conventional physiotherapy - a randomized control trial. Int J Neurorehabilitation. 2017;4(6):301; doi: 10.4172/2376 $-0281.1000301$.

20. Moczek K, Gawlik K, Rosołek B. The relationship between physical activity and the prevalence of disabilities caused by back pain in men over 60 years of age. Postep Rehabil.2018;32(4):29-35; doi:10.5114/areh.2018.83392.

21. Lim JU, Lee JH, Kim JS, Hwang YI, Kim T-H, Lim SY, et al. Comparison of World Health Organization and AsiaPacific body mass index classifications in COPD patients. Int J Chron Obstruct Pulmon Dis. 2017;12:24652475; doi: 10.2147/COPD.S141295.

22. Kanno H, Ozawa H, Koizumi Y, Morozumi N, Aizawa T, Kusakabe T, et al. Dynamic change of dural sac crosssectional area in axial loaded magnetic resonance imaging correlates with the severity of clinical symptoms in patients with lumbar spinal canal stenosis. Spine. 2012; 37(3):207-213; doi: 10.1097/BRS.0b013e3182134e73.

23. Mathur R, Swaminathan S, The Core Advisory Group. National ethical guidelines for biomedical \& health research involving human participants, 2017: a commentary. Indian J Med Res. 2018;148(3):279-283; doi: 10.4103/0971-5916.245303.

24. Portney LG, Watkins MP. Correlation. In: Portney LG, Watkins MP, Foundations of clinical research: applications to practice, $3^{\text {rd }}$ ed. Philadelphia: F.A. Davis Company; 2015; 523-537. 